\title{
Review
}

\section{Structure and function in the p53 family}

\author{
CH Arrowsmith ${ }^{\star 1}$ \\ ${ }^{1}$ Ontario Cancer Institute and Department of Medical Biophysics, University of \\ Toronto, 610 University Ave., Toronto, Ontario, Canada M5G 2M9 \\ * Corresponding author: CH Arrowsmith, Ontario Cancer Institute and \\ Department of Medical Biophysics, University of Toronto, 610 University Ave., \\ Toronto, Ontario, Canada M5G 2M9. Tel: 416946 2017; Fax: 416946 6529; \\ E-mail: carrow@oci.utoronto.ca
}

Received 15.9.99; revised 15.10.99; accepted 21.10.99

Edited by R Knight

\begin{abstract}
The recent discovery of several $\mathrm{p} 53$ homologs has uncovered a 553 superfamily of transcription factors that can trigger cell cycle arrest and apoptosis. The challenge now is to understand the similarities and differences between family members especially in terms of their regulation and potential for physical or genetic interactions with one another. This review summarizes recent progress in understanding the structure-function relationship within the p53 family. The new family members, $p 63$ and p73, have an additional conserved domain at their $\mathrm{C}$-termini which may have a regulatory function. The structure of this domain (a SAM domain) suggests that it is a protein-protein interaction module that may be involved in developmental processes. The oligomerization domains of p53 family members, while conserved in sequence and three-dimensional structure do not interact appreciably with other family members, but do mediate interactions between the multiple splice variants from an individual gene.
\end{abstract}

Keywords: tumor suppressor; SAM domain; oligomerization; protein-protein interaction

Abbreviations: SAM, sterile alpha motif; NMR, nuclear magnetic resonance; Eph, ephrin

\section{Introduction}

$p 53$ is a tumor suppressor gene and the most frequent site of genetic alterations found in human cancers. ${ }^{1,2}$ The p53 protein is a transcription factor that regulates the expression of a wide variety of genes involved in cell cycle arrest and apoptosis in response to genotoxic or cellular stress (reviewed in references ${ }^{3-5}$ ). Growth arrest or cell death prevents damaged DNA from being replicated and suggests a role for p53 in maintaining the integrity of the genome. ${ }^{6}$ This activity is central to its role as a tumor suppressor and also of major importance in the response of many cancers to conventional therapies which trigger apoptosis by damaging DNA. Inactivation of p53 through either deletion, mutation or interaction with cellular or viral proteins is a key step in the development of over half of all human cancers. ${ }^{1,2,7}$ Recently, two new p53-like proteins have been identified, $p 73^{8}$ and $p 63$ (also called KET, p51, and p40), ${ }^{9-12}$ suggestive of a larger superfamily of p53-like proteins which share similar, but not identical structures and functions. Below is a review of our current knowledge of the three-dimensional structures of p63 and p73, and the structure-function relationship within the p53 superfamily.

\section{Modular architecture}

Like many transcription factors, p53-like proteins are modular molecules with a conserved transcriptional activation domain, DNA-binding domain and an oligomerization domain that mediates tetramerization (Figure 1). Both p63 and p73 have an additional C-terminal extension not found in p53. This Cterminal region is subject to alternative splicing resulting in multiple isoforms, designated $p 73 \alpha-\zeta, 8,13,14$ and $p 63 \alpha-\gamma^{9}$ with different biological activities. P63 also has isoforms which lack the $\mathrm{N}$-terminal activation domain $(\Delta \mathrm{Np} 63)$ due to an alternative transcription start site. ${ }^{9}$

Although no structural information has been reported for the activation or DNA-binding domains of p63 and p73, the significant levels of sequence identity of these proteins relative to p53 suggest that the three-dimensional structures of the individual homologous domains will be very similar. For example, the oncoprotein, MDM2, binds to the N-terminal transcriptional activation domain of p53 and negatively regulates the activity and stability of p53 as part of an auto-inhibitory feedback loop. ${ }^{3,4}$ Residues 17-27 of p53 (TSESDLWEKLㄴP) which interact with MDM2 are

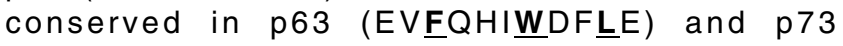
(TTEEHLWSSLEE). This sequence in p53 forms an amphipathic $\alpha$-helix in which the underlined/bold residues bind in a hydrophobic pocket of the $\mathrm{N}$-terminal domain of MDM2. ${ }^{15}$ The conservation of these critical interacting residues suggests that both p63 and p73 will also bind to MDM2 via a similar mechanism. Indeed, direct binding of p73 to MDM2 has been observed. ${ }^{16,43}$ Similarly, the high level of sequence identity among the DNA binding domains and the overlapping DNA-binding specificity suggests that p63 and p73 interact with DNA in a manner similar to that reported by Cho et al, for p53. ${ }^{17}$ Nevertheless, there is ample evidence that $\mathrm{p} 53$ and $\mathrm{p} 73$ differentially regulate transcription of p53-target genes. ${ }^{13,22,23}$ While part of these differences may be mediated by regions in the C-terminus of each protein (see below), it is also very likely that differences in sequence in the DNA-binding domain mediate subtle differences in DNA-binding specificity. 


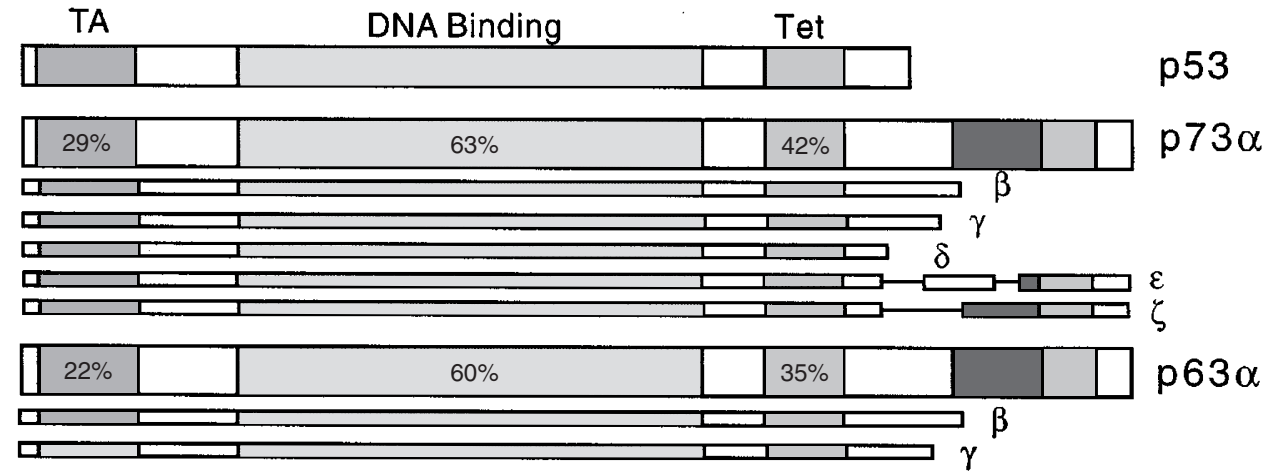

Figure 1 Gene structure of the p53 family members with splice forms indicated $(\alpha-\zeta)$ and regions of sequence and structural homology shaded; TA, transactivation domain; Tet, tetramerization domain. The per cent identity at the amino acid level relative to p53 is indicated for the transactivation, DNA binding and tetramerization domains. The darkest shaded section of the C-terminus of p63 and p73 corresponds to the SAM domain

Further structural and/or biochemical studies that directly measure DNA-binding specificity (as opposed to transcriptional activity) will be required to uncover to what extent there is discrimination among DNA target sequences within the p53 family.

\section{Oligomerization}

Many families of transcription factors exhibit combinatorial homotypic and heterotypic interactions via conserved oligomerization or protein-protein interaction domains. We have shown that like p53, the individual oligomerization domains of p63 and p73 form homotetramers. ${ }^{18}$ Because the tetramerization domains of p63 and p73 are present in all splice forms (Figure 1) it is expected that in vivo each protein will be a mixed homo-oligomer composed of a statistical distribution of those splice forms available in a given cell type. Oligomerization-mediated interactions among splice variants is supported by the fact that the DNA-binding mutant, $\mathrm{p} 73 \alpha(\mathrm{R} 293 \mathrm{H})$, can inhibit transcriptional transactivation by wild-type $\mathrm{p} 73 \alpha$ when the two proteins are coexpressed in SK-N-AS cells which lack expression of endogenous p73 protein. ${ }^{18}$ This is consistent with the report by Yang et $a l,{ }^{9}$ in which a GST-tagged p63 $\gamma$ was able to interact with several other p63 isoforms. Both these observations likely reflect interactions mediated by the oligomerization domain of p73 and p63, respectively, and likely have important implications for their activity in cells expressing multiple isoforms.

In addition to homo-oligomerization, it is of interest to know whether p53 family members can hetero-oligomerize. The fact that the tetramerization domains of $p 63$ and $p 73$ share 35 and $42 \%$ sequence identity with $p 53$, respectively, raises the possibility of physical interactions between p53 family members as is often the case within other families of transcription factors such as the homeodomain proteins ${ }^{19}$ or the Myc superfamily. ${ }^{20}$ In vitro experiments with recombinant oligomerization domains ${ }^{18,21}$ clearly support the notion that p53 does not heterooligomerize with p63 or p73, even though all three proteins likely homo-oligomerize via a similar three-dimensional structure (Figure 2). Similarly, in vivo experiments showed

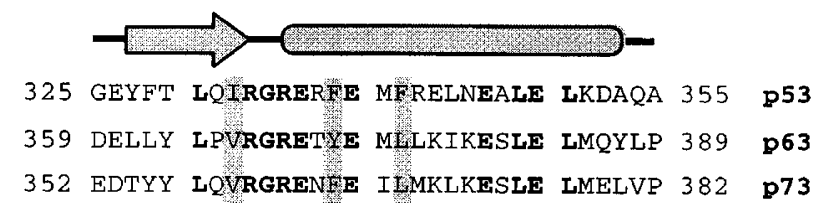

Figure 2 A sequence alignment of the tetramerization domains of p53, p63 and p73. The secondary structure 40 of this domain in p53 is indicated across the top. The arrow represents a $\beta$-sheet and the cylinder corresponds to an $\alpha$ helix. The high sequence similarity is highly predictive of very similar threedimensional tertiary structures for this domain in all three proteins. In particular, the crucial sequence, RGR, that defines the tight turn between the $\beta$-sheet and $\alpha$-helix is absolutely conserved. Hydrophobic residues along the sheet and helix are responsible for the association of four molecules into homo tetramers. Three key residues identified by Mateu and Fersht ${ }^{21}$ as being important for oligomerization specificity are shaded

that the DNA-binding mutant, p53(R273H), did not interfere with transcriptional transactivation by $\mathrm{p} 73 \alpha$, and $\mathrm{p} 73 \alpha(\mathrm{R} 293 \mathrm{H})$ did not interfere with transactivation by wild-type p53. ${ }^{18}$ These results suggest that transactivation-deficient isoforms of p63 or p73 (such as N-terminally truncated proteins ${ }^{9}$ ) will not hetero-oligomerize with wildtype p53 thereby affecting p53 transcriptional activity. Lack of hetero-oligomerization by p53 may have important practical consequences. For example, the use of p73 in gene therapy instead of p53 to trigger apoptosis in cancer cells, should overcome the dominant negative effect of mutant p53. An alternative therapeutic strategy could be to up-regulate the endogenous transcriptionally active isoforms of p63 or p73 in cancers with mutant p53.

A lack of hetero-oligomerization between p53 and p73 suggest that the accumulation of mutant p53, as is frequently observed in human cancers, will likely not interfere with p63 or p73 transcriptional activity. However, it has been reported that mutant p53 (R175H and R248W) but not wild-type p53 could co-immunoprecipitate with p73 and that mutant p53, but not wild-type p53 could reduce the transcriptional and apoptotic activities of p73 in $\mathrm{H} 1299$ cells. ${ }^{22}$ Because these interactions were not observed for wild-type p53 they are unlikely to be mediated by the oligomerization domains of each protein and may involve some unknown feature of these p53 mutants. 
In contrast to $\mathrm{p} 53$, we have shown that p63 can heterooligomerize with p73 via its tetramerization domain. ${ }^{18}$ This interaction appears to be weak because it is only observable with an excess of one of the two proteins, and dominant negative p73 mutants were not fully able to inhibit p63 transactivation even in tenfold excess. The fact that the p63 and p73 oligomerization domains are more closely related to each other (58\% identity) than either is to p53 (35 and 42\% identity, respectively) may explain why p63 and p73 are able to interact to some extent. These data raise the possibility of cooperative, or antagonistic

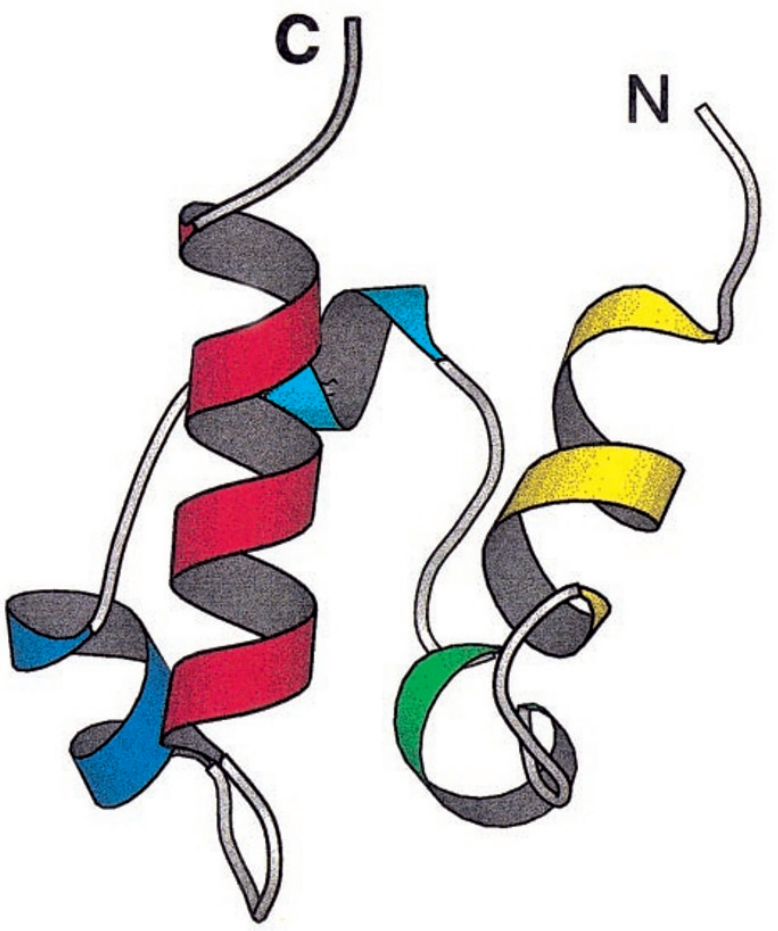

Figure 3 A ribbon diagram representing the backbone of the SAM domain of p73 (PDB accession number 1cok). Helices are colored from $\mathrm{N}$ - to C-terminus: yellow, green, cyan, blue and red. The figure was generated with Molscript ${ }^{41}$ interactions between p63 and p73 if they are co-expressed, or mis-expressed in the same tissue. However, coexpression of both proteins has yet to be demonstrated, and further data on the expression patterns of both p63 and p73 will be needed in order to better understand the functional relationship, if any, between these two proteins.

\section{A C-terminal SAM domain in p63 $\alpha$ and $\mathbf{p 7 3} \alpha$}

Both $p 63 \alpha$, and $p 73 \alpha$ contain a conserved C-terminal region of approximately 100 residues not found in human p53 (Figure 1 ). There is evidence that p63 and p73 isoforms that differ in this C-terminal region have differential transcriptional and biological properties. For example, p63 $\gamma$ was able to transactivate a p53 reporter gene to a level approximately $80 \%$ of that of wild-type p53, whereas p63 $\alpha$ was unable to activate the same reporter gene. ${ }^{9}$ Differences in the degree of transactivation by $\mathrm{p} 73$ isotypes have also been reported. $^{13,22,23}$ These data suggest that the C-terminal region of $p 73 \alpha$ and $p 63 \alpha$ may have regulatory properties that modulate the transcriptional activity of these proteins and therefore may contribute to the distinctive biological properties of these proteins relative to $\mathrm{p} 53$.

We have used nuclear magnetic resonance (NMR) spectroscopy to determine the three-dimensional solution structure of this domain from human $p 73 .{ }^{24}$ Residues $555-$ 600 appear to be unstructured, but residues $487-554$ form a 5-helix bundle (Figure 3) with marked similarity to the structures of SAM (sterile alpha motif) domains from Ephrin receptor tyrosine kinases. ${ }^{25-27} \mathrm{~A}$ distant relationship at the sequence level $(15-17 \%$ identity) has been previously noted between p53-like proteins and SAM domains. ${ }^{28-30}$ It was suggested that p73 and its homologs may be involved in protein-protein interactions involved in developmental regulation of transcription. ${ }^{29}$ The structural similarity between the p73 SAM domain and the Eph receptor SAM domains strengthens this argument. The key hydrophobic residues that form the hydrophobic core of p73(487-554) (Figures 3 and 4) align with positions of hydrophobic residues in a consensus sequence that was derived from a multiple alignment of more than 40 members of the SAM

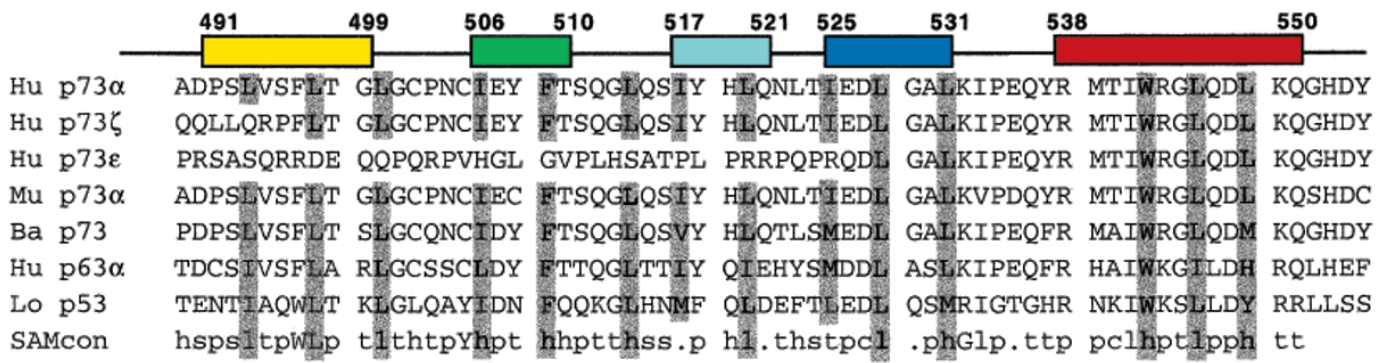

Figure 4 Sequence alignment of the SAM domains of human p73 isoforms ( $\mathrm{Hu}$ ), Murine p73 (Mu), p73 from Barbus barbus (Ba), human p63 $\alpha$, and a protein originally annotated as squid (Loligo) $p 53^{42}$ (Lo). The SAM domain consensus sequence derived from multiple sequence alignment of more than 60 SAM domains ${ }^{28}$ is shown along the lower line. Uppercase letters are the one-letter amino acid codes. Lowercase letters are: $h$, hydrophobic; I, aliphatic; $p$, polar; $c$, charged; s, small; t, turnlike (tiny or polar). The helices of the SAM domain of $p 73 \alpha^{24}$ are shown across the top with colors corresponding to those shown in Figure 3 . Residue numbers of the $\mathrm{N}$ - and $\mathrm{C}$-termini of each helix are indicated. The key hydrophobic residues that interact to form the hydrophobic core of the p73 SAM domain are shaded in gray for p73 and other proteins in which these hydrophobic residues are identical or of similar amino acid type. While only L497 is absolutely conserved with the SAM consensus sequence, most other hydrophobic core residues correspond to a hydrophobic or aliphatic residue in the SAM consensus 
superfamily. ${ }^{28}$ However, the pattern of surface residues of p73(487-554) is most similar to that of p63 and unrelated to that of the Eph receptors. Thus, the p73/p63 C-terminal domains may constitute a unique subclass of human SAMlike domains.

SAM domains are protein-protein interaction modules found in a variety of cytoplasmic signaling proteins as well as several transcriptional regulatory proteins. The majority of these proteins play an important role in developmental regulation. ${ }^{28}$ The most notable type of protein-protein interaction identified for SAM domains to date, is that of homo- and hetero-oligomerization among similar SAM domains. The SAM domains of the Eph receptors have been shown to homo-dimerize in a crystal environment and weakly dimerize in solution. ${ }^{25,26}$ This SAM-mediated dimerization has been proposed as a mechanism for Eph receptor activation. The pointed domain (a SAM-like domain) of the ETS transcription factor, TEL, can also self-associate, ${ }^{31}$ and has been found fused to other signaling and regulatory proteins in many leukemias, resulting in constitutive activation of these fusion proteins via dimerization. ${ }^{32,33}$ Finally, members of the polycomb group of homeotic transcriptional regulators form homoand hetero-oligomers via their SAM domains. ${ }^{34,35}$

We found no evidence of homo-oligomerization of the SAM domain of p73 using three different techniques: NMR spectroscopy, equilibrium sedimentation and size exclusion chromatography. ${ }^{24}$ This result is not surprising, since both p63 and p73 have separate, very efficient tetramerization domains (see above). We also tested whether the SAM-like domains of p63 and p73 could interact with one another, since each is the other's closest relative in terms of sequence identity and surface properties. Again, no interactions were observed using either $\mathrm{Ni}^{2+}$ affinity chromatography of mixtures of the differentially hexahistidine-tagged domains, or size exclusion chromatography of mixtures of the two domains. ${ }^{24}$ Thus, if the p73/p63 SAM domain is involved in protein-protein interactions, the partner proteins must be either a more distantly related SAM domain, or quite possibly a non-SAM domain protein(s).

The SAM domain of p73 and p63 is present in the $\alpha$, but not the $\beta, \gamma$ (or $\delta$ ) isoforms of both proteins and only partially present in the $\varepsilon$ and $\zeta$ isoforms of p73 (Figures 1 and 4$)$. The $\zeta$ isoform contains most of the SAM domain sequence, but lacks a crucial hydrophobic residue (L493 in $\mathrm{p} 73 \alpha$ ) that contributes to the folding and stability of this domain. Thus, it is not clear whether the $\zeta$ isoform contains a folded, functional SAM domain. The $\varepsilon$ isoform lacks the first three and a half helices and therefore is unlikely to be folded and may not be functional. There is mounting evidence that the various $\mathrm{C}$-terminal isoforms of p63 and p73 may have differential transcriptional and biological activities. ${ }^{9,13,14,22,23}$ P53 contains a C-terminal regulatory domain ${ }^{36}$ but this region is the most divergent in sequence relative to $p 63$ and $p 73$. Therefore, the unique C-termini of $p 63 \alpha$ and $p 73 \alpha$ may also play a regulatory role via protein-protein interactions modulated by alternative splicing. Studies of gene-targeted mice have shown that unlike p53, p63 and p73 play an important role in development. ${ }^{10,37-39}$ Because SAM domains are most often found in proteins involved in development or differentiation $^{28}$ it is tempting to hypothesize that the SAM domains of p63 and p73 may also mediate such processes.

\section{Acknowledgements}

Research in the author's lab is supported by the National Cancer Institute of Canada with funds from the Terry Fox Run, the Medical Research Council of Canada and the Leukemia Research Fund of Canada. Several passages describing the structure of the p73 SAM domain were reproduced from reference 24 with permission from EMBO and Oxford University Press.

\section{References}

1. Hollstein M, Shomer B, Greenblatt M, Soussi T, Hovig E, Montesano Rand Harris CC (1996) Database of p53 gene somatic mutations in human tumors and cell lines. Nucl. Acids Res. 24: 141-146

2. Hollstein M, Sidransky D, Vogelstein B and Harris CC (1991) p53 mutations in human cancer. Science 253: 49-53

3. Ko LJ and Prives C (1996) p53: puzzle and paradigm. Genes Dev. 10: 1054 1072

4. Levine AJ (1997) p53, the Cellular Gatekeeper for Growth and Division. Cell 88: $323-331$

5. Donehower LA and Bradley A (1993) The tumor suppressor p53. Biochimica et Biophysica Acta 1155: 181-205

6. Lane DP (1992) p53, guardian of the genome. Nature 358: 15-16

7. Nigro JM, Baker SJ, Preisinger AC, JessupJM, Hostetter R, Cleary K, Bigner SH, Davidson N, Baylin S, Devilee P, Glover T, Collins FS, Weston A, Modali R, Harris $\mathrm{CC}$ and Vogelstein $\mathrm{B}$ (1989) Mutations in the p53 gene occur in diverse human tumour types. Nature 342: 705-708

8. Kaghad M, BonnetH, Yang A, Creancier L, Biscan J-C, Valent A, Minty A, Chalon P, Lelias J-M, Dumont X, Ferrera P, McKeon F and Caput D (1997) Monoallelically expressed gene related to $p 53$ at 1 p36, a region frequently deleted in neuroblastoma and other cancers. Cell 809-819

9. Yang A, Kaghad M, Wang Y, GillettE, Fleming MD, Dotsch V, Andrews NC, Caput D and McKeon F (1998) p63, a p53 homolog at 3q27-29, encodes multiple products with transactivating, death-inducing, and dominant-negative activities. Mol. Cell 2: $305-316$

10. Schmale $H$ and Bamberger $C$ (1997) A novel protein with strong homology to the tumor suppressor p53. Oncogene 15: 1363-1367

11. Osada M, Ohba M, Kawahara C, Ishioka C, Kanamaru R, Katoh I, Ikawa $Y$, Nimura Y, Nakagawara A, Obinata M and lkawa S (1998) Cloning and functional analysis of human p51, which structurally and functionally resembles p53. Nature Medicine 4: 839-843

12. Trink B, Okami K, Wu L, Sriuranpong V, Jen J and Sidransky D (1998) A new human p53 homologue. Nature Medicine 4: 747-748

13. De Laurenzi V, Costanzo A, Barcaroli D, Terrinoni A, Falco M, AnnicchiaricoPetruzzelli M, Levrero M and Melino G (1998) Two new p73 splice variants, gamma and delta, with different transcriptional activity. J. Exp. Med. 188: 17631768

14. De Laurenzi V, Catani M, Costanzo A, Terrinoni A, Corazzari M, Levrero M, Knight Rand Melino G(1999) Additional complexity in p73: induction by mitogens in lymphoid cells and identification of two new splicing variants epsilon and zeta. Cell Death Differ. 6: 389-390

15. Kussie PH, Gorina S, Marechal B, Elenbaas B, Moreau J, Levine AJ and Pavletich NP (1996) Crystal structure of the MDM2 oncoprotein bound to the transactivation domain of the p53 tumor suppressor. Science 274: 948-953

16. Balint $E$, Bates $S$ and Vousden $\mathrm{KH}$ (1999) Mdm2 binds to p73 without targeting degradation. Oncogene 18: 3923-3929

17. Cho Y, Gorina S, Jeffery P and Pavletich N (1994) Crystal structure of p53 tumor suppressor-DNA complex: understanding tumorigenic mutations. Science 265 : $346-355$ 
18. Davison TD, Vagner C, Kaghad M, Ayed A, Caput D and Arrowsmith CH (1999) p73 and 63 are homo-tetramers capable of weak hetero-typic interactions with each other but not p53. J. Biol. Chem. 274: 18709-18714

19. Sharkey M, Graba Y and Scott MP (1997) Hox genes in evolution: protein surfaces and paralog groups. Trends In Genetics 13: 145-155

20. Schreiber-Agus N, Alland L, Muhle R, Goltz J, Chen K, Stevens L, Stein D and DePinho RA (1997) A biochemical and biological analysis of Myc superfamily interactions. Curr. Topics in Microbiol. \& Immun. 224: 159-168

21. Mateu MG and Fersht AR (1999) Mutually compensatory mutations during evolution of the tetramerization domain of tumor suppressor p53 lead to impaired hetero-oligomerization. Proc. Natl. Acad. Sci. USA 96: 3595-3599

22. Di Como CJ, Gaiddon C and Prives C (1999) p73 function is inhibited by tumorderived p53 mutants in mammalian cells. Mol. Cell. Biol. 19: 1438-1449

23. Zhu J, Jiang J, Zhou W and Chen X (1998) The potential tumor suppressor p73 differentially regulates cellular p53 target genes. Cancer Res. 58: 5061-5065

24. Chi S-W, Ayed A and Arrowsmith CH (1999) Solution structure of a conserved Cterminal domain of $p 73$ with structural homology to the SAM domain. EMBO J. 18: $4438-4445$

25. Stapleton D, Balan I, Pawson T and Sicheri F (1999) The crystal structure of an Eph receptor SAM domain reveals a mechanism for modular dimerization. Nat. Struct. Biol. 6: 44-49

26. Thanos CD, Goodwill KE and Bowie JU (1999) Oligomeric structure of the human EphB2 receptor SAM domain. Science 283: 833-836

27. Smalla M, Schmieder P, Kelly M, terLaak A, Krause G, Ball L, Wahl M, Bork P and Oschkinat $\mathrm{H}$ (1999) Solution structure of the receptor tyrosine kinase EphB2 SAM domain and identification of two distincthomotypic interaction sites. Protein Sci. 10: $1954-1961$

28. Schultz J, Ponting CP, Hofmann $K$ and Bork $P$ (1997) SAM as a protein interaction domain involved in developmental regulation. Protein Sci. 6: 249253

29. Bork P and Koonin EV (1998) Predicting functions from protein sequences where are the bottlenecks? Nat. Genet. 18: 313-318

30. Thanos CD and Bowie JU (1999) p53 Family members p63 and p73 are SAM domain-containing proteins. Protein Sci. 8: 1708-1710

31. Jousset C, Carron C, Boureux A, Quang CT, Oury C, Dusanter-FourtI, Charon M, Levin J, Bernard O and Ghysdael J (1997) A domain of TEL conserved in a subset of ETS proteins defines a specific oligomerization interface essential to the mitogenic properties of the TEL-PDGFR beta oncoprotein. EMBO J. 16: 69-82
32. Golub TR, Barker GF, Lovett M and Gilliland DG (1994) Fusion of PDGF receptor beta to a novel ets-like gene, tel, in chronic myelomonocytic leukemia with $\mathrm{t}(5 ; 12)$ chromosomal translocation. Cell 77: 307-316

33. Golub TR (1997) TEL gene rearrangements in myeloid malignancy. Hematol. Oncol. Clin. North. Am. 11: 1207-1220

34. Peterson AJ, Kyba M, Bornemann D, Morgan K, Brock HW and Simon J (1997) A domain shared by the Polycomb group proteins $\mathrm{Scm}$ and ph mediates heterotypic and homotypic interactions. Mol. Cell. Biol. 17: 6683-6692

35. Kyba M and Brock HW (1998) The SAM domain of polyhomeotic, RAE28, and scm mediates specific interactions through conserved residues. Dev. Genet. 22: $74-84$

36. Hupp TR, Meek DW, Midgley CA and Lane DP (1992) Regulation of the specific DNA binding function of p53. Cell 71: $875-886$

37. Almog $N$ and Rotter $V$ (1998) An insight into the life of p53: a protein coping with many functions! Review of the 9th p53 Workshop, Crete, May 9-13, 1998. Biochim. Biophys. Acta 1378: R43-54

38. Mills AA, Zheng B, Wang XJ, Vogel H, Roop DR and Bradley A (1999) p63 is ap53 homologue required for limb and epidermal morphogenesis. Nature 398: $708-$ 713

39. Yang A, Schweitzer R, Sun D, Kaghad M, Walker N, Bronson RT, Tabin C, Sharpe A, Caput D, Crum C and McKeon F (1999) p63 is essential for regenerative proliferation in limb, craniofacial and epithelial development. Nature 398: 714-718

40. Lee W, Harvey TS, Yin Y, Yau P, Litchfield D and Arrowsmith CH (1994) Solution structure of the tetrameric minimum transforming domain of p53. Nat. Struct. Biol. $1: 877-890$

41. Kraulis PJ (1991) MOLSCRIPT: a program to produce both detailed and schematic plots of protein structures. J. Appl. Crystallogr. 24: 946-950

42. Ishioka C, Shimodaira H, Englert C, Shimada A, Osada M, Jia J-Q, Suzuki T, Gamo M and Kanamaru R (1997) Oligomerization is not essential for growth suppression by p53 in p53-deficient Osteosarcoma Saos-2 cells. Biochem. Biophys. Res. Com. 232: 54-60

43. Zeng X, Chen L, Jost CA, Maya R, Keller D, Wang X, Kaelin JrWG, Oren M, Chen $\mathrm{J}$ and Lu H (1999) MDM2 suppresses p73 function without promoting p73 degradation. Mol. Cell. Biol. 19: 3257-3266 